\title{
Super-resolution techniques in aerial images for remote sensing
}

\section{Victor Lima*, Renato Lopes}

\begin{abstract}
Super-resolution algorithms, specially when applied in remote sensing, are widely used for many purposes as defense and agricultural research. Classical super-resolution algorithms use multiple low-resolution (LR) images of the target to extract information and use them to build a new image of superior resolution. The LR sources must differ in the sub-pixel range. In contrast, this paper applies an iterative process, using a single LR image to produce a high resolution image.
\end{abstract}

\section{Key words:}

Super-resolution, Remote sensing, Aerial image.

\section{Introduction}

The first method ${ }^{1}$ builds an HR grid with twice the resolution. All the pixels are labeled $A, B, C$ or $D$ and each type comes from a different image. Pixels from the first LR image rest on the A-type pixels of the HR grid and receive no further treatment. $B, C$ and D-types pixels are determined taking the average of the surrounding pixels in the corresponding LR image. The mapping of LR pixels to $B$-type is shown in image 1 below.
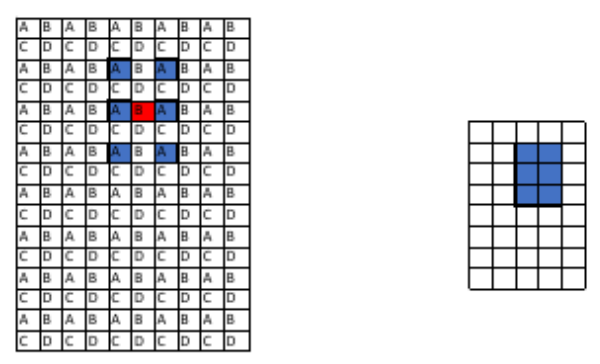

Image 1. B-type pixel mapping

The other pixel types are treated similarly. This method assumes all images are georeferenced. That means the actual geographic feature lying in center of the pixel is the same in all images thus the variation of the images comes from the different time of acquisition.

The other method is called Iterative-Back Procedure $(\mathrm{IBP})^{2}$ and uses a single image. The first step is to interpolate the LR image replicating each pixel 4 times. The new HR image is down-sampled and compared to the original LR image. The error is interpolated and summed to the HR image until the error is low enough. The process also considers distortion and noise when down-sampling. Since the images used in this work were already treated for these effects, the Gaussian filter and noise introduction was removed from the algorithm.

\section{Results and Discussion}

To study the different methods of super-resolution we used a $40 \times 40$ px image with spacial resolution of 1 $\mathrm{KM} / \mathrm{pixel}$ obtained with the Center for Agricultural Research of the University of Campinas (CEPAGRI/UNICAMP). The images depicts the city of Ihabela in the state of São Paulo - Brazil. One of them is shown in image 2 .
To implement the fist method 4 images taken in may/2011 were used. All images were already georeferenced with 1 px precision. The result from both methods are shown in image 2 below.
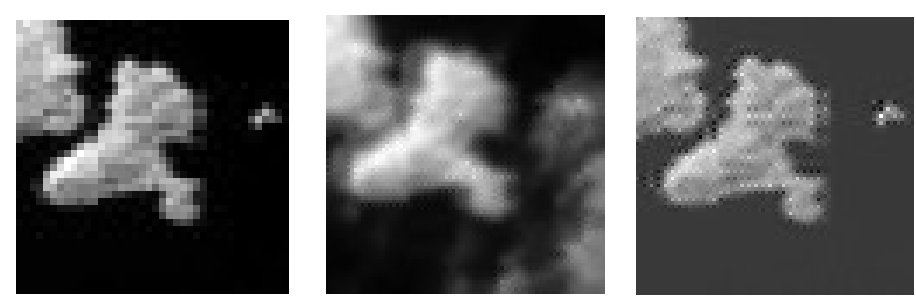

Image 2. In order: Original image, Classical SR image and IBP image.

We can observe that both increased resolution images show an increase in edges definition. The Classical SR image is vulnerable to contamination of source images, though the clouds appearing in the bottom right corner of one of the sources were attenuated. The ghost-like effect was caused by georreferencing errors within the $1 \mathrm{px}$ precision. The IBP process is more robust since it depends on a single image to work but it shows a grainy resulting image.

\section{Conclusions}

The study of these methods contributed to detect strong and weak points of both methods. Both methods show an improvement in features detection.

The classic SR method demands an image positioning error correction to eliminate the blurring.

The IBP method shows an increased feature definition but a contrast enhancement is in need. Both methods show interesting performance and can be applied to remote sensing applications when the requirements above are met.

\section{Acknowledgment}

This project is funded by CNPq/PIBIC program and the images were cede by CEPAGRI/UNICAMP.

\footnotetext{
${ }^{1}$ J. Yang e T. Huang, Super-Resolution Imaging, CRC Press, 2010.

R. Fernandez-Beltran, P. Latorre-Carmona e F. Pla, Single-frame superresolution in remote sensing: a practical overview, 2017.
} 